\title{
Retrospective longitudinal study on canine and feline parvovirus infections in Al Ain, United Arab Emirates
}

\author{
Shamma Abdulla Al Eissaee ${ }^{1}$, Khaja Mohteshamuddin ${ }^{1 *}$, Zaib Mahel ${ }^{2}$, Gobena Ameni ${ }^{183,}$ \\ ${ }^{1}$ Department of Veterinary Medicine, College of Food and Agriculture, United Arab Emirates University, PO Box 15551, Al Ain, Abu Dhabi, \\ UAE, ${ }^{2}$ Paws \& Claws Veterinary Clinic, Al Bawadi Market Enterprise, Al Ain, Abu Dhabi, UAE, ${ }^{3}$ Aklilu Lemma Institute of Pathobiology, Addis \\ Ababa University, PO box 1176, Addis Ababa, Ethiopia
}

\section{A B S T R A C T}

\begin{abstract}
Background: There is a lack of epidemiological data on parvovirus infections in cats and dogs in the Al Ain, United Arab Emirates (UAE). Objective: To conduct retrospective longitudinal study on parvovirus infections in cats and dogs treated at the Claws and Paws Veterinary Clinic in Al Ain, UAE. Methods: Data on clinical examination and laboratory tests were extracted in March 2020 retrospectively from case records of cats and dogs treated at Paws \& Claws Veterinary Clinic in Al Ain between February 2019 to March 2020. Results: Of the total 2247 cases of small animals treated at Claws and Paws clinic for different health problems between February 2019 and March 2020, 68 were parvovirus infection cases constituting 3\% (95\% confidence interval, $\mathrm{Cl}: 2.3 \%, 3.8 \%$ ) of the total small animal cases treated at the Clinic during the indicated time. Seventy-two percent of the parvovirus cases were feline parvovirus (FPV) cases (feline panleukopenia) while the remaining $28 \%$ of the cases were canine parvovirus (CPV) cases. The difference in the proportions of CPV and FPV was significant $\left(\chi^{2}=26.14 ; p<0.001\right)$. Overall, the number of cases was low during most months of the year; but increased in November and December 2019 reaching its peak in January 2020 after which it declined. Significant (Cochran's $Q$ test $=141.54$; $p<0.001$ ) difference was observed in the proportions of animals exhibited different clinical signs. Conclusion: This study indicated the endemicity of CPV and FPV infections in Al Ain. Additionally, increase in number of cases was observed between November January, suggesting seasonality of the epidemics requiring for vaccination prior to the epidemic period.
\end{abstract}

Keywords: Canine parvovirus; epidemiology; clinical data; Feline panleukopenia; United Arab Emirates

\section{INTRODUCTION}

Canine parvovirus (CPV) and feline parvovirus (FPV) infections cause severe gastroenteritis, which is characterized by diarrhea, vomiting, leukopenia and dehydration. The two infections occur worldwide affecting domestic cats and dogs as well as wild carnivores causing fatalities in young ones (Sun et al., 2019; Van et al., 2019; Jenkins et al., 2020 \& Qi et al., 2020). The disease in cats is named as feline panleukopenia or feline parvo while it is termed as canine parvovirus infection in dogs. Nonetheless, the two diseases have similar pathophysiological and clinical characteristics (Scott, 1987). Moreover, according to the current taxonomy, CPV and FPV are classified as a single taxonomic entity (Tattersal, 2006). This is because of the evolvement of CPV from FPV in late 1970s through acquiring five to six amino acid changes in the capsid protein gene as a result of crossing species barrier (Truyen, 1999). The first CPV that has been evolved from FPV has been designated as CPV-2 so as to differentiate it from canine parvovirus type 1 (CPV-1), which causes neonatal death in dogs and re-classified under the Genus Bocavirus in which bovine parvovirus and human parvovirus are grouped (Tattersal, 2005).

Following its evolvement from FPV, CPV-2 was changed to new subtypes named CPV-2a and CPV-2b, which can infect cats and cause clinical signs that cannot be differentiated from the clinical signs that are caused by FPV infection (Truyen et al., 1995; Truyen et al., 1996; Ikeda et al., 2000 \& Mochizuki et al., 1996) although CPV-2 could not infect cats. Furthermore, the emergence of a new subtype which has been designated as CPV-2c occurred in Italy in 2000 and this subtype rapidly spread to other countries.

In general, the although FPV has not undergone significant change in the antigenic and biological properties (Shackelton

\footnotetext{
*Corresponding author:

Khaja Mohteshamuddin, Department of Veterinary Medicine, College of Food and Agriculture, United Arab Emirates University, PO Box 15551, Al Ain, Abu Dhabi, UAE. E-mail: drkhaja707@uaeu.ac.ae
} 
et al., 2005), CPV has progressively evolved (Shackelton et al., 2005 \& Decaro et al., 2009). Subsequently, the new variants of CPV are characterized by pathogenicity in dogs and are able to infect and cause disease in cats (Decaro et al., 2008). Furthermore, epidemiological surveys indicated that the newest subtype, which is CPV-2c, has become prevalent in many countries causing a severe disease. Both FPV and CPV are transmitted by fecal-oral route and primary spread by contact with infected body fluids, feces or other fomites as well as fleas. The two viruses are highly resistant to external environment and can survive up to one year in contaminated material (Truyen et al., 2009) and this nature of the virus makes it best fitted to spread during the whole year.

Van et al., (2019) studied the parvoviral infections in Australia, New Zealand \& the UAE and in UAE Fourteen cases of Feline parvovirus were diagnosed in domestic crossbreds with positive faecal CPV antigen test results. The animals were strays or strays that had been recently adopted, and all were unvaccinated, except an 18-monthold cat that had received a primary course of kitten vaccination and a booster vaccination at one year. Clinical signs reported were vomiting, diarrhoea and lethargy, and the median age at diagnosis was 4 months. All cats were treated, and outcomes were known in 12 cases, of which 8 died or were euthanised, and 4 survived.

According to our observation to small animal clinics in $\mathrm{Al}$ Ain City, parvovirus infection is prevailing and its incidence rate in $\mathrm{Al}$ Ain City is probably increasing. To the best of our knowledge there is no epidemiological study that has been conducted on parvovirus infection in small animals in the $\mathrm{Al}$ Ain City so far. Therefore, the objective of paper was to conduct a cross-sectional study through the extraction of retrospective data on parvovirus infection in small animals at Paws \& Claws Veterinary Clinic in Al Ain in UAE.

\section{MATERIALS AND METHODS}

\section{Study design}

The study design was a retrospective longitudinal study which was conducted by extracting retrospective data from the case records of the small animals treated in the at the Claws and Paws Clinic between February 2019 and March 2020. Clinical data were extracted and used for analysis.

\section{Study setting}

The study was conducted at the Paws and Claws Veterinary Clinic, Al Bawadi District in Al Ain City, UAE. The Clinic was selected mainly because of its good clinical record keeping and the large number of its clients as compared to the other small animal clinics in the $\mathrm{Al}$ Ain City.

\section{Inclusion criteria}

The case records of all the dogs and cats treated at Claws and Paws between February 2019 and March 2020 were eligible for the screening. Thus, of the total 2247 cases of dogs and cats treated at Claws and Paws clinic for different diseases during this specified time, 68 parvovirus infection cases were identified and included in the study. The algorithms used for the selection of the cases were reports of clinical signs that show gastroenteritis such as diarrhea, vomiting, and dehydration (Sun et al., 2019; Van et al., 2019; Jenkins et al., 2020 \& Qi et al., 2020). In addition, fever, lethargy and inappetence were considered (Sun et al., 2019; Van et al., 2019; Jenkins et al., 2020 \& Qi et al., 2020). On top of these clinical signs, laboratory diagnosis of CPV was made using IDEXX SNAP CPV antigen test kit that was applied to fecal samples of dogs (IDEXX, 2020) while laboratory diagnosis of FPV was based on the detection of the antigen of FPV test in the feces or vomitus of cats by SensPERT FPV Ag test kit (VetAll Laboratories, 2020).

\section{Study animals}

Animals included in the study were cats and dogs that diagnosed with either FPV and CPV, respectively between February 2019 and March 2020. Accordingly, during this specified period 68 cats and dogs were treated in the Clinic and thus, this study used the clinical data of these animals.

\section{Sample size \& sampling}

As the study was dependent on the number of cases that visit the Clinic, determination of sample size using statistical parameters was not required. Similarly, due to the same reason, probability sampling techniques could not be considered. Retrospectively, recorded cases of cats and dogs with parvovirus infections were screened from the case records, and used for data extraction. Data were collected on the number of cases, clinical signs, treatment, species and sex of the cases.

\section{Diagnosis of parvovirus infections in cats and dogs}

The veterinarians in clinic use clinical and laboratory examination for the diagnosis of parvovirus infections in cats and dogs. The important clinical signs, which were considered were diarrhea (watery, hemorrhagic or mucoid), vomiting, anorexia, dehydration, fever, lethargy and inappetence. Exercise intolerance was also reported in some cases. Laboratory diagnosis of CPV infection was made using IDEXX SNAP CPV antigen test kit and applied to fecal samples of dogs (IDEXX, 2020). The laboratory diagnosis of FPV was based on the detection of the antigen of FPV test in the feces or vomitus of cats by SensPERT FPV Ag test kit (VetAll Laboratories, 2020).

\section{Statistical analysis}

The data were entered into spread sheets of Excel and analyzed. Descriptive statistics was used to express the 
results. Two proportions were compared using a chi-square $\left(\chi^{2}\right)$ test. Comparisons of more than two proportions was made using non-parametric Cochran's Q test. Proportions with none overlapping 95\% confidence intervals (CIs) were considered as significantly different. Additionally, when proportions were compared using $\chi^{2}$ test and the $\mathrm{p}$ value was $<0.05$, the compared proportions were considered to be statistically different.

\section{RESULTS}

Canine and feline parvovirus cases treated at Paws and Claws Veterinary Clinic during February 2019 and March 2020

Of the total 2247 small animals treated at Claws and Paws clinic for different disease between February 2019 and March 2020, 68 were new cases of parvovirus infection cases. Thus, the percentage of parvovirus infection was 3\% (95\% CI: $2.3 \%, 3.8 \%$ ) in small animal cases treated the indicated specific time. Majority $(72 \%)$ of the cases were FPV while the remaining 28\% were CPV (Table 1). The difference between the number of CPV and FPV cases was statistically $\left(\chi^{2}=26.14 ; \mathrm{p}<0.001\right)$ significant. However, there was no significant difference $\left(\chi^{2}=0.00 ; \mathrm{p}>0.05\right)$ in percentage of parvovirus infection between female and male animals.

Figure 1 shows the number of cases presented at the Clinic between February 2019 and March 2020. Overall, a low

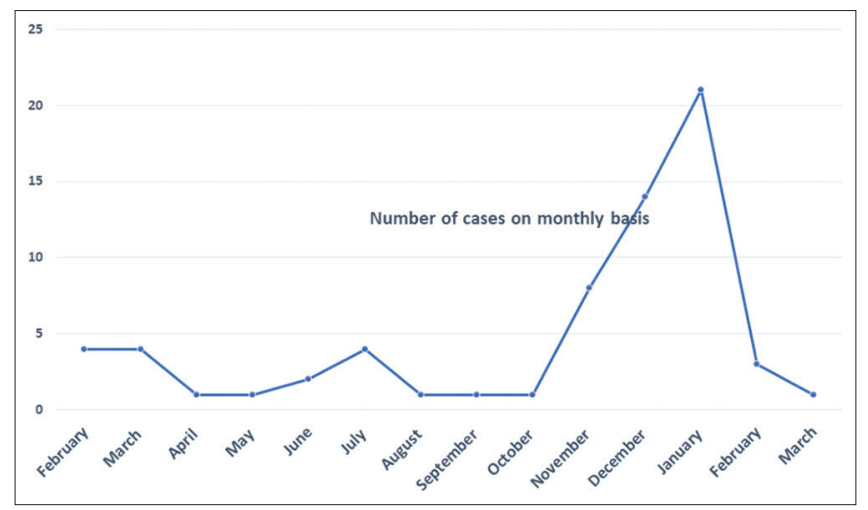

Fig 1. Number of cases parvovirus infections in small animals that were presented at Paws \& Claws Veterinary Clinic during 2019. The number of was low during the most of the months of the 2019; but the number of cases started to rise in November 2019 and reaching its peak in January 2020 after which it declined rapidly. number of parvovirus infection cases were recorded until the last two months of 2019. However, at the end of the Year from November to December and then peaking in January 2020. Thereafter, the number of cases decreased dramatically in February and March 2020

\section{Clinical signs exhibited by parvovirus infection cases of cats and dogs}

The number of daily admitted cases was presented in supplementary file and the data include the dates of when the animal was administered to the Clinic, and the species and sex of the infected animal. It also shows the major clinical signs. The most frequently exhibited clinical signs were diarrhea, fever and vomiting, each of them manifested by $44.8 \%, 43.3 \%$ and $22.4 \%$ of the cases, respectively (Table 2). Figure 2 illustrates the clinical signs exhibited by parvovirus-infected case of dog, which included bloody diarrhea, lethargy and depression. Cochran's Q test (141.54) showed the presence of difference $(p<0.001)$ in proportions of animals exhibited diarrhea, fever, vomiting, lethargy, inappetence, dehydration, constipation, and salivation. The difference in the proportions of animals exhibiting different clinical signs could be pinpointed by scrutinizing overlapping of the $95 \%$ CI of the proportions. No statistically significant difference was observed among proportions of animals showed diarrhea, fever, and vomiting while these proportions were statistically varied from the proportions of animals with lethargy, inappetence, dehydration, constipation, and salivation.

\section{Types of treatment given to Parvovirus-infected small animals at Paws \& Claws Veterinary Clinic}

All of the clinical cases required isolation and hospitalization at the Clinic. The treatment constituted of fluid therapy and antibiotics. Ringer lactate solution was administered using intravenous route while Marbofloxacin was administered either intravenously or subcutaneously at a dose rate of $2.5 \mathrm{mg} / \mathrm{kg}$ once daily for about a week. Vetoquino $\mathbb{}$ Ornipural® Solution (https://www.primoveterinary.net/) combination of vitamins, minerals, electrolytes and amino acids was also given to all cases subcutaneously $2-5 \mathrm{ml}$ depending on the size of the animal according to the recommendation of the manufacturer (Vetoquino, 2017). For boosting the immunity of the animals $1 \mathrm{ml}$ of Zylexis (Hyperdrug.co.uk, https://www.hyperdrug.co.uk/) was

Table 1: Association of species and sex of the small animals with Parvovirus infection

\begin{tabular}{lcccccc}
\hline & Canine & Feline & Total & Percentage & Chi-square $\left(\chi^{2}\right)$ & P-value \\
\hline Male & 11 & 23 & 34 & 50 & 0.00 & \\
Female & 8 & 26 & 34 & 50 & \\
Total & 19 & 49 & 68 & & \\
Percentage & $28 \%$ & $72 \%$ & & & \\
Chi-square $\left(\chi^{2}\right)$ & & 26.14 & & & & \\
P value & & $\mathrm{p}<0.001$ & & & \\
\hline
\end{tabular}


Table 2: Proportions the major clinical signs of recorded clinical sign from 68 animals

\begin{tabular}{lccc}
\hline Clinical sign & $\begin{array}{c}\text { No of exhibited } \\
\text { animal }\end{array}$ & $\begin{array}{c}\text { \% of exhibited } \\
\text { animal }\end{array}$ & $95 \% \mathbf{~ C l}$ \\
\hline Diarrhea & 30 & 44.8 & $33.6-57.4$ \\
Vomiting & 15 & 22.4 & $13.1-34.2$ \\
Fever & 29 & 43.3 & $31.2-56.0$ \\
Lethargy & 8 & 12.9 & $5.3-22.2$ \\
Inappetence & 9 & 13.4 & $6.3-2.4$ \\
Dehydration & 4 & 6.0 & $1.7-1.5$ \\
Constipation & 1 & 1.5 & $0-8.0$ \\
Salivation & 1 & 1.5 & $0-8.0$ \\
\hline
\end{tabular}

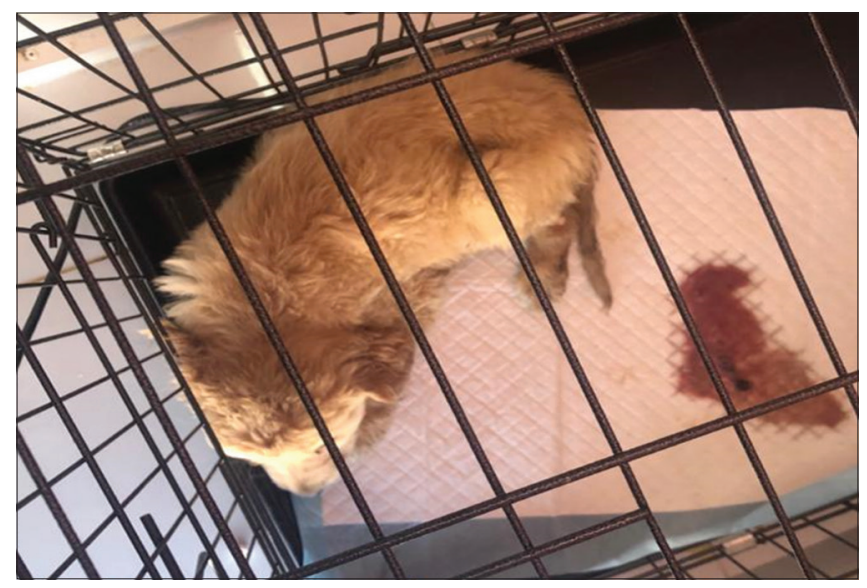

Fig 2. A canine parvovirus case hospitalized at the Paws and Claws Veterinary Clinic in Al Ain City. The puppy was seriously ill, clinically was characterized by depression, lethargy and bloody diarrhea.

administered subcutaneously regardless of the weight of the animal on the first day of arrival, then repeated after 48 hours and then the final dose after 72 hours according to the manufacturer ${ }^{18}$.

\section{DISCUSSION}

A retrospective longitudinal study was conducted by extracting the clinical data of cat and dog cases of parvovirus infections retrospectively from Paws and Claws Veterinary Clinic in Al Ain City between February 2019 and March 2020. A total of 68 parvovirus infection cases were treated during the indicated period of time and majority of the cases were FPV infection cases while CPV infection cases was significantly lower. The number of cases was stable during all months of the year; but started to rise in November 2019 and reached peak in January 2020 after which it declined.

The explanation for observing higher percentage of FPV infection as compared to CPV infection could be the existence of real difference between cats and dogs in susceptibility to parvovirus infection. Supporting this observation, earlier study reported the occurrence of inapparent and subclinical CPV infections in pups with maternally derived antibody and adult dogs (IDEXX, 2020). However, the mortality can reach $70 \%$ in pups with no maternal antibody while it is usually less than $1 \%$ in adult dogs (VetAll Laboratories, 2020). On the other hand, the mortality rate of FPV infection ranges from $25-90 \%$ in acutely infected cats and can reach 100\% in per-acute infections (Vetoquino, 2017). Kittens within 12 weeks of age were observed to have the highest morbidity and mortality rates (Hyperdrug, 1998; Decaro et al., 2005). The incidence rate of the virus is usually higher in catteries, shelters, kennels and pet shops (Decaro et al., 2012; Stuetzer \& Hartman, 2014). The other possible explanation for the observed difference in the proportions of CPV and FPV infections could be due to unequal number of cats and dogs kept in $\mathrm{Al}$ Ain City. If the larger proportion of the households are keeping cats, then large number of FPV infection cases are obviously expected to visit the veterinary clinics. Additionally, the difference in the vaccination coverage between dogs and cats could lead to such differences in the incidence of parvovirus infections in the two species of small animals. On the other hand, this study did not show difference in the proportion female and male cases of parvovirus infection. Similar to this observation, previous studies recorded the no effect of sex on the occurrence of parvovirus infection in small animals (Addie et al., 1998; Cave at al., 2002). The major clinical signs recoded were diarrhea, fever and vomiting, which have also been reported earlier in parvovirus infections in cats and dogs (Qi et al., 2020; Kruse et al., 2010).

The number of cases was stable during all months of the year but started to rise in November December of 2019 and January 2020. In agreement with the observation of this study, Van Brussel et al. reported exactly the same seasonal distribution of Australian and New Zealand FPV infection cases in which the number of cases started to rise in November and reached peak in January. Furthermore, seasonal variation in the incidence of CPV infection was reported earlier (Qi et al., 2020). November, December and January are cold months with intermittent rain in UAE and rise in the incidence of CPV and FPV infections could be that the wet environment facilitates fecal contamination and dirt in the pens and surroundings of the houses and which in turn facilitate the transmission of the viruses. The other possible reason could be the stress that is caused by the cold weather, which in turn weakens the protective immunity of the animals and makes them more susceptible to the infection. On other hand, a study conducted in Nigeria indicated that the occurrence of large number of cases of parvovirus infections in dry season (Kumar \& Nandini, 2010). In both cases, since the virus is resistant to external environment and can survive up to 1 year in infected organic material (Truyen et al., 2009) it is possible that the cat/dog owners can carry the virus into their houses on their hands, shoes or clothing and infect their pets indoors (Behera et al., 2015). Additional 
epidemiological data should be collected to generate reliable knowledge on the effect of season on the epidemiology of parvovirus infections in cats and dogs in UAE.

Although there is no study conducted on parvovirus infections in small animals in UAE, a study conducted on simultaneous outbreaks of FPV infections between 2014 and 2018 in Australia, New Zealand and UAE indicated that each of outbreak was caused by a distinct FPV; but the viruses from the UAE outbreak formed a lineage of unknown origin (Van et al., 2019) requiring for further investigation. Similarly, the different subtypes of CPV have been reported from different countries including China and India (Wang et al., 2016). In China, examination of 43 fecal samples of dogs using PCR resulted in identification 18 CPV-2a, 7 CPV-2b, and 18 CPV-2c subtypes (Awad \& Atallah, 2018). While in India, molecular characterization of $78 \mathrm{CPV}$ isolates lead to the identification of $27 \mathrm{CPV}-2 \mathrm{a}$, 39 CPV-2b, 12 CPV-2c subtypes (Stuetzer \& Hartman, 2014). In addition, the occurrence of FPV infection in cats was further confirmed using molecular identification in India (Parthiban et al., 2014; Hasan et al., 2017). There could be similar distribution of FPV and CPV subtypes in UAE because of strong connection between the Indian and Emirati people. However, there is no data on the distribution of the different subtypes of CPV and FPV infections in UAE so far. In addition, although there are reports on the infection of cats by CPV from different countries especially from South Asian countries like Vietnam and Taiwan where $80 \%$ of the diseased cats were infected with CPV (Daodu \& Ajiboye, 2018), it is not known if cats in UAE are infected with CPV or not.

\section{LIMITATION}

This study was conducted on secondary data and as a result had inherited limitations. The first limitation was the failure to include the clinical data of all the 68 cases since such data were not recorded in the case record. Besides, the treatment outcome of each case could not be obtained from the case record. The second limitation was the failure to estimate the incidence rates of the two infections as it was not possible to obtain data on the populations of dog and cat in the study District. The third limitation was failure to identify the strains of the parvovirus viruses circulating in cat and dog populations. In this connection, it would have been important to detect if both cats and dogs were infected by the same or different viruses.

\section{CONCLUSION}

This preliminary study showed the significance of parvovirus infection in small animals in $\mathrm{Al}$ Ain City. However, additional studies on a larger scale are required in order to establish the real epidemiological picture of the disease including its molecular epidemiology. Parallel to these, regular vaccination prior to the outbreak season is recommended to prevent parvovirus infections in both cats and dogs.

\section{ABBREVIATIONS}

CPV: canine parvovirus; CPV-1: canine parvovirus type 1; CPV-2: canine parvovirus type 2; CPV-2a: canine parvovirus type 2 subtype a; CPV-2b: canine parvovirus type 2 subtype b; CPV-2c: canine parvovirus type 2 subtype c; FPV: feline parvovirus; FP: feline panleukopenia; UAE: United Arab Emirates

\section{ACKNOWLEDGMENTS}

The authors thank the personnel working in the Paws and Claws Veterinary Clinic for their support during data extraction. The parents of the first author are appreciated for supporting the first author during data collection and beyond.

\section{Funding}

No funding was allocated for the study. All co-authors used their own resources in undertaking this study.

\section{COMPETING INTERESTS}

Dr Mahel is the owner of the Clinic and a veterinarian in her Clinic. But did not know that we would do this study when she was treating these animals. In addition, she was not involved in the extraction of data from the record and also has not participated in data analysis.

\section{Ethical approval}

The study was approved by the Council of the Department of Veterinary Medicine of the College of Food and Agriculture of the UAE University as a Senior student research Project of the first author. Additionally, the study obtained support by the Paws and Claws Clinic. Otherwise, no other ethical approval was required.

\section{Authors' contributions}

SAE performed data extraction and drafted the manuscript. KM assisted in designing the study and edited the manuscript, ZM supported in data extraction and edited the manuscript. GA designed the study, analyzed the data and critically edited the manuscript.

\section{REFERENCES}

Addie, D.D., Toth, S., Thompson, H., Greenwood, N., and Addie, D. D., S. Toth, H. Thompson, N. Greenwood and J. O. Jarrett. 1998. Detection of feline parvovirus in dying 
pedigree kittens. Vet. Rec. 142: 353-356.

Awad, R., W. Khalil and A. Attallah. 2018. Epidemiology and diagnosis of feline panleukopenia virus in Egypt: Clinical and molecular diagnosis in cats. Vet. World. 11: 578-584.

Behera, M., S. Panda, P. Sahoo, A. Acharya, R. Patra, S. Das and S. Pati. 2015. Epidemiological study of canine parvovirus infection in and around Bhubaneswar, Odisha, India. Vet. World. 8: 33-37.

Cave, T. A., H. Thompson, S. W. J. Reid, D. R. Hodgson and D. D. Addie. 2002. Kitten mortality in the United Kingdom: A retrospective analysis of 274 histopathological examinations (1986 to 2000). Vet. Rec. 151: 497-501.

Daodu, O. and B. Ajiboye. 2018. Retrospective study of canine parvoviral enteritis in Ilorin, North Central, Nigeria. J. Vet. Sci. 13: $32-38$.

Decaro, N. and C. Buonavoglia. 2012. Canine parvovirus a review of epidemiological and diagnostic aspects, with emphasis on Type 2. Vet. Microbiol. 155: 1-12.

Decaro, N., M. Campolo, C. Desario, G. Elia, V. Martella, E. Lorusso and C. Buonavoglia. 2005. Maternally-derived antibodies in pups and protection from canine parvovirus infection. Biologicals. 33:259-265.

Decaro, N., C. Desario, A. Miccolupo, M. Campolo, A. Parisi, V. Martella, F. Amorisco, M. S. Lucente, A. Lavazza and C. Buonavoglia. 2008. Genetic analysis of feline panleukopenia viruses from cats with gastroenteritis. J. Gen. Virol. 89: 22902298.

Decaro, N., C. Desario, A. Parisi, V. Martella, A. Lorusso, A. Miccolupo, V. Mari, M. L. Colaianni, A. Cavalli, L. Di Trani and C. Buonavoglia. 2009. Genetic analysis of canine parvovirus Type 2c. Virology. 385: 5-10.

Hasan, M., M. Jalal, M. Bayzid, M. Sharif and M. Masuduzzaman. 2017. A comparative study on canine parvovirus infection of dogs in Bangladesh and India. Bangladesh J. Vet. Med. 14: 237-241.

Hyperdrug. 1998. Canine, Feline, Equine and Pigeon Pharmacies. Available from: https://www.hyperdrug.co.uk. [Last accessed on 2020 Dec 28].

IDEXX. 2020. SNAP Parvo. . Available from: https://www.idexx. com/en/veterinary/snap-tests/snap-parvo-test/@2019. [Last accessed on 2020 Dec 28].

Ikeda, Y., M. Mochizuki, R. Naito, K. Nakamura, T. Miyazawa, T. Mikan and E. Takahashi. 2000. Predominance of canine parvovirus (CPV) in unvaccinated cat population and emergence of new antigenic types of CPVs in cats (rapid communication). Virology. 278: $13-19$.

Jenkins, E., C. Davis, M. Carrai, M. P. Ward, S. O'Keeffe, M. van Boeijen, L. Beveridge, C. Desario, C. Buonavoglia, J. A. Beatty, N. Decaro and V. R. Barrs. 2020. Feline parvovirus seroprevalence is high in domestic cats from disease outbreak and non-outbreak regions in Australia. Viruses. 12: 320.

Kruse, B., S. Unterer, K. Horlacher, C. Sauter-Louis and K. Hartmann. 2010. Prognostic factors in cats with feline panleukopenis. J. Vet. Intern. Med. 24: 1271-1276.

Kumar, M. and S. Nandi. 2010. Molecular typing of canine parvovirus variants by polymerase chain reaction and restriction enzyme analysis. Transbound. Emerg. Dis. 57: 458-463.

Mochizuki, M., M. Horiuchi, H. Hiragi, M. C. San Gabriel, N. Yasuda and T. Uno. 1996. Isolation of canine parvovirus from a cat manifesting clinical signs of feline panleukopenia. J. Clin. Microbiol. 34: 2101-2105.

Parthiban, M., K. S. Aarthi, M. Balagangatharathilagar and K. Kumanan. 2014. Evidence of feline panleukopenia infection in cats in India. Virus Dis. 25: 497-499.

Qi, S., J. Zhao, D. Guo and D. Sun. 2020. A mini-review on the epidemiology of canine parvovirus in China. Front. Vet. Sci. 7: 1-10.

Scott, F. W. 1987. Viral diseases. Panleukopenia. In: J. Hotzworth (Ed.), Diseases of the Cat: Medicine and Surgery, WB Saunders Co., Philadelphia, PA, USA, pp. 182-193.

Shackelton, L. A., C. R. Parrish, U. Truyen and E. C. Holmes. 2005. High rate of viral evolution associated with the emergence of carnivore parvo virus. Proc. Natl. Acad. Sci. 102: 379-384.

Stuetzer, B. and K. Hartman. 2014. Feline parvovirus infection and associated diseases (Review). Vet. J. 201: 150-155.

Sun, Y., Y. Cheng, P. Lin, H. Zhang, L. Yi, M. Tong, Z. Coa, S. Li, S. Cheng and J. Wang. 2019. Simultaneous detection and differentiation of canine parvovirus and feline parvovirus by high resolution melting analysis. BMC Vet. Res. 15: 141. Tattersall, P. (2006) The evolution of parvovirus taxonomy. In: J. Kerr, S. F. Cotmore, M. E. Bloom, R. M. Linden and C. R. Parrish (Eds.), Oxford University Press, New York, USA, pp. 5-14.

Tattersall, P., M. Bergoin, M. E. Bloom, K. E. Brown, R. M. Linden, N. Muzyczka, C. R. Parrish and P. Tijssen. 2005. Family Parvoviridae In: C. M. Fauquet, M. A. Mayo, J. Maniloff, U. Desselberger and L. A. Ball (Eds.), Virus Taxonomy: $8^{\text {th }}$ Report of the International Committee on Taxonomy of Viruses. Elsevier Academic Press, Cambridge, Massachusetts.

Truyen, U. 1999. Emergence and recent evolution of canine parvovirus. Vet. Microbiol. 69(1-2):47-50

Truyen, U., D. Addie, S. Belak, C. Boucraut-Baralon, H. Egberink, T. Frymus, T. Gruffydd-Jones, K. Hartman, M. J. Housie, A. Loiret, H. Lutz, F. Marsilio, M. G. Pennisi, A. D. Radford, E. Thiry and M. C. Horzinek. 2009. Feline panleukopenia. ABCD guidelines on prevention and management. J. Feline Med. Surg. 11: 538-546.

Truyen, U., J. F. Evermann, E. Vieler and C. R. Parrish. 1996. Evolution of canine parvovirus involved loss and gain of feline host range (a short communication). Virology. 215: 186-189.

Truyen, U., A. Gruenberg, S. F. Chang, B. Obermaier, P. Veijalainen and C. R. Parrish. 1995. Evolution of the feline-subgroup parvoviruses and the control of canine host range in vivo. J. Virol. 69: 4702-4710.

Van Brussel, K., M. Carrai, C. Lin, M. Kelman, L. Setyo, D. Aberdein, J. Brailey, M. Lawler, S. Maher, I. Plaganyi, E. Lewis, A. Hawkswell, A. B. Allison, J. Meers, V. Martella, J. A. Beatty, E. C. Holmes, N. Decaro and V. R. Barrs. 2019. Distinct lineages of feline parvovirus associated with epizootic outbreaks in Australia, New Zealand and the United Arab Emirates. Viruses. 11: 1155.

VetAll Laboratories. 2020. Veterinary Company Specialized in Animal Diseases Available from: http://www.vetall.com/en/Gyeonggi-do. [Last accessed on 2020 Dec 28].

Vetoquino. (2017) ®Ornipural®Solution. Primo Veterinary Medicines Trading LLC. Available from: https://www.primoveterinary.net/ Copyright@2017. [Last accessed on 2020 Dec 28].

Wang, J., P. Lin, H. Zhao, Y. Cheng, Z. Jiang, H. Zhu and S. Cheng. 2016. Continuing evolution of canine parvovirus in China: Isolation of novel variants with an Ala5Gly mutation in the VP2 protein. Infect. Genet. Evol. 38: 73-78. 